\title{
Psychotherapie im höheren Lebensalter in der Psychotherapieausbildung
}

\section{Eine Bestandsaufnahme}

\author{
Tobias Becker ${ }^{1}$, Franziska Martin², Gabriele Wilz², Anne Katrin Risch², Eva-Marie Kessler ${ }^{3}$ \\ und Simon Forstmeier ${ }^{1}$ \\ 'Institut für Psychologie, Universität Siegen \\ ${ }^{2}$ Institut für Psychologie, Friedrich-Schiller-Universität Jena \\ ${ }^{3}$ Department Psychologie, MSB Medical School Berlin Hochschule für Gesundheit und Medizin, Berlin
}

\begin{abstract}
Zusammenfassung: Theoretischer Hintergrund: Psychotherapie im höheren Lebensalter (PihL) gewinnt in Deutschland in den letzten Jahrzehnten zunehmend an Bedeutung. Dennoch ist dieses Feld trotz des großen Bedarfs weiterhin unterrepräsentiert. Fragestellung: Wie werden angehende Psychotherapeut_innen aktuell in Bezug auf die psychotherapeutische Behandlung älterer Menschen ausgebildet? Methode: Sämtliche Ausbildungsinstitute in Deutschland mit dem Schwerpunkt Verhaltenstherapie sowie Dozierende mit Seminaren zu PihL wurden im Jahr 2018 zu einer Befragung eingeladen. Ergebnisse: Von 48 Instituten boten zum Zeitpunkt der Befragung 64.6\% gerontopsychlogische Unterrichtsstunden $(\mathrm{M}=13.03 \mathrm{~h})$ an $(2.2 \%$ des Theorieunterrichts). In den ausfindig gemachten Seminaren zeigen sich zu einem großen Teil die von Knight et al. (2009) vorgeschlagenen Themenschwerpunkte von allgemeinen und pathologischen Alterungsprozessen über diagnostische Besonderheiten bis hin zu konkreten Interventionsmethoden. Diskussion: Sowohl die Weiterbildungsstudiengänge als auch die neuen psychotherapeutischen Bachelor- und Masterstudiengänge sollten mehr Inhalte zum Thema PihL ähnlich wie die hier ausfindig gemachten Seminare berücksichtigen. Eine Orientierung für eine Basiswissensvermittlung bieten die vorgeschlagenen Themenschwerpunkte.
\end{abstract}

Schlüsselwörter: Psychotherapie, höheres Lebensalter, Psychotherapeutenausbildung, Gerontopsychologie, Gerontologie

Psychotherapy with Older Adults in Psychotherapy Training. A Survey

Abstract: Background: Over the last decades, psychotherapy with older adults has become increasingly important in Germany. Yet, despite the great need, this field remains underresearched. Question: How are psychotherapists in training currently being educated to provide psychotherapy to older adults? Methods: In 2018, psychotherapy training institutions in Germany focusing on behavioral therapy as well as lecturers providing seminars on psychotherapy with older adults were asked to complete a survey. Results: $64.6 \%$ of the 48 institutions queried offered geropsychology classes ( $M=13.03 \mathrm{~h} ; 2.2 \%$ of curriculum). The seminars identified contained largely thematic foci proposed by Knight et al. (2009), ranging from general and pathological aging processes to diagnostic features and interventions. Discussion: Psychotherapy training programs for graduate students as well as the newly founded psychotherapy bachelor and master programs for undergraduates should consider introducing more geropsychology content like the seminars identified. The proposed thematic foci provide an orientation for basic knowledge transfer.

Keywords: psychotherapy, older adults, clinical training, geropsychology, gerontology

In der ersten Hälfte des 20. Jahrhunderts gab es praktisch keine Psychotherapie für Menschen im höheren Lebensalter. Wenn überhaupt, dann wurde das höhere Lebensalter eher als Kontraindikation für Psychotherapie betrachtet (Kessler, 2021). In den 1960er Jahren setzte sich in den USA die neu gegründete ,Boston Society for Gerontologic Psychiatry' erstmals mit psychotherapeutischen
Fragen im Alter auseinander. Wegbereiter in Deutschland waren in den 1970er Jahren beispielsweise der Psychoanalytiker Hartmut Radebold, sowie Ursula Lehr mit ihrer verhaltenstherapeutisch begründeten Interventionsgerontologie (Kessler \& Peters, 2017). In den 1980er Jahren fand die Psychotherapie mit älteren Menschen durch die Verhaltenstherapie ein größeres Forschungsecho. Als 
Beispiele sind das von Gallagher und Thompson $1981 \mathrm{pu}-$ blizierte Behandlungsmanual "Depression in the elderly“, sowie das 1986 erschienene Lehrbuch von Bob Knight „Psychotherapy with older adults“ anzuführen (Kessler \& Tegeler, 2018). Die ,American Psychological Association formulierte 2003 erstmalig eigene ,Guidelines for Psychotherapy with older adults' (American Psychological Association, 2014). Die Tatsache, dass ein Grundkonsens unter Experten gefunden wurde, markiert, dass sich das Forschungsfeld seit den 2000er Jahren klar etabliert hat.

Die Notwendigkeit einer intensiveren Befassung mit dem Thema ergibt sich insbesondere aufgrund des demographischen Wandels. In den nächsten zwei Jahrzehnten wird in Deutschland die Gruppe der über 67-Jährigen um 5 bis 6 Millionen auf über 20.9 Millionen Menschen ansteigen. Die Kohorte der hochaltrigen Menschen (über 80 Jahre) wird sich im Vergleich zu 2018 (5.4 Millionen) im Jahr 2050 fast verdoppelt haben und schätzungsweise zwischen 8.9 und 10.5 Millionen liegen (Statistisches Bundesamt, 2019). Zudem liegt in der Altenbevölkerung eine vergleichsweise hohe Selbstmordrate vor (Statistisches Bundesamt, 2020), weshalb sich auch hieraus die Notwendigkeit verstärkter psychotherapeutischer Unterstützung ergibt (Wächtler, 2009).

Zwar liegt die 12-Monats-Prävalenz psychischer Störungen im höheren Lebensalter (65-79 Jahre) mit 20.3\% unter der Gesamtprävalenz (27.7\%) aller Altersgruppen (Jacobi et al., 2014), dennoch fällt die psychotherapeutische Versorgung für ältere Menschen in Deutschland immer noch ausgesprochen defizitär aus. Die schlechte Versorgungssituation lässt sich am besten für ambulante Psychotherapie bei Diagnose Major Depression belegen, da hierzu für Deutschland aktuelle Behandlungsprävalenzen vorliegen. Nach Analysen vertragsärztlicher Abrechnungsdaten über alle Krankenkassen hinweg befanden sich im Jahr 2016 von den depressiv diagnostizierten Versicherten über 65 Jahre etwa $5 \%$, bei den hochaltrigen Patient_innen weniger als $1 \%$ in ambulanter psychotherapeutischer Behandlung (Kessler \& Tegeler, 2018). Im Vergleich zu der Versorgungssituation bei den jungen Erwachsenen (etwa $20 \%$ ) zeigt sich bei den Patient_innen im höheren Lebensalter demnach eine erhebliche Unterversorgung.

Dass für die Behandlung älterer Patient_innen die Einstellung von Psychotherapeut_innen gegenüber dem höheren Lebensalter bedeutsam erscheint, konnte in einer experimentellen Fallvignettenstudie mit 114 angehenden Psychotherapeut_innen verdeutlicht werden (Kessler \& Blachetta, 2020). Diese bekamen entweder die Fallbeschreibung eines 86-jährigen, depressiven Patienten zu lesen oder die identische Version eines 52-jährigen, depressiven Patienten, bei dem lediglich das Alter und altersrelevante Wörter (bspw. Haarfarbe) verjüngt wurden.
Es zeigte sich, dass Therapeut_innen gegenüber älteren Patient_innen in Bezug auf deren Therapiefähigkeit und -erfolg pessimistischer eingestellt waren. Zudem schätzten sie ihre eigene Behandlungskompetenz in Bezug auf die Behandlung älterer Patient_innen signifikant geringer ein (Kessler \& Blachetta, 2020). Eine andere Studie, in der 119 niedergelassene Psychotherapeut_innen in Berlin befragt wurden, fand einen positiven Zusammenhang zwischen den spezifischen Kenntnissen in der psychotherapeutischen Arbeit mit älteren Menschen und der Anzahl behandelter älterer Patient_innen (Peters, Jeschke \& Peters, 2013). In einer Befragung von 431 angehenden Therapeut_innen in Australien zeigte sich zudem, dass das Interesse an der Behandlung älterer Menschen mit praktischen, klinischen Erfahrungen in diesem Bereich positiv zusammenhängt (Koder \& Helmes, 2008). Zusammenfassend bedarf es offenbar theoretischer und praktischer gerontopsychologischer Qualifikation, um auch die Bereitschaft von Psychotherapeut_innen für die Behandlung älterer Menschen zu steigern.

Gleichzeitig steht die Wirksamkeit von Psychotherapie im höheren Lebensalter heute außer Frage. So liegen positive Wirkungsnachweise für (generalisierte) Angsterkrankungen (Ayers, Sorrell, Thorp \& Wetherell, 2007; Boerner, 2004; Gould, Coulson \& Howard, 2012; Hall, Kellett, Berrios, Bains \& Scott, 2016), depressive Erkrankungen (Cuijpers, Karyotaki, Pot, Park \& Reynolds, 2014; Gühne, Luppa, König, Hautzinger \& Riedel-Heller, 2014; Pinquart, Duberstein \& Lyness, 2007), Insomnie (Nau, McCrae, Cook \& Lichstein, 2005; Richter, Kellner, Milosheva \& Fronhofen, 2020) oder posttraumatische Belastungsstörung (Böttche, Kuwert \& Knaevelsrud, 2012) vor. Bei somatoformen Störungen zeigten sich in einer Pilotstudie erste ermutigende Befunde hinsichtlich der Abnahme von Schmerzintensität (Verdurmen, Videler, Kamperman, Khasho \& Feltz-Cornelis, 2017). Auch bei leichtgradigen Demenzen erweist sich die kognitive Verhaltenstherapie als wirksam (Forstmeier \& Roth, 2018).

Knight, Karel, Hinrichsen, Qualls und Duffy (2009) legten auf Grundlage einer Zusammenführung von Ergebnissen einer Fachkonferenz aus dem Jahr 2006 (Pikes Peak conference) das sogenannte "Pikes Peak Model“ vor, in dem zentrale Kompetenzen für die Behandlung von Patient_innen im höheren Lebensalter abgebildet sind. Dabei weisen die AutorInnen darauf hin, dass nicht jede/r behandelnde Therapeut_in, die/der ältere Patient_innen behandelt, solch umfassende Kenntnisse benötige. Vielmehr würden die vorgelegten Kompetenzen dann umso wichtiger, je spezifischer und komplexer altersspezifische Prozesse die psychische Erkrankung und damit die Therapie beeinflussen. Dieses Modell teilt sich in die drei Kernbereiche „Einstellungen“, „Basiswissen“ und „Kompetenzen" einhergehend mit verschiedenen Domänen auf 
Tabelle 1. Vorschlag von Hinrichsen et al. (2018) zur Verteilung von 15 Unterrichtsstunden basierend auf 5 Wissens/Einstellungs-Domänen des Pikes Peak Model (Knight et al., 2009). Domäne 1 entspricht dem Kernbereich „Einstellungen“. Die Domänen 2 bis 5 gehören zum Kernbereich „Basiswissen“

\begin{tabular}{llc}
\hline & Domäne & Stunden \\
\hline 1 & Einstellung gegenüber älteren Menschen und dem Alter & 1.5 \\
2 & Allgemeines Wissen über Alterungsprozesse & 2.5 \\
3 & Grundlagenwissen klinischer Praxis mit Älteren & 3 \\
4 & Grundlagenwissen Diagnostik bei Älteren & 3.5 \\
5 & Interventionen, Beratung und andere Dienstleistungen & 3.5 \\
$+\quad$ Vertiefung in einer der oben genannten Themenschwerpunkte, & 1 \\
& je nach spezifischer Subgruppe oder Setting des/r Psychologen/in & \\
\hline
\end{tabular}

(Knight et al., 2009). Basierend auf den Bereichen „Einstellungen“ und „Basiswissen“ des Pikes Peak Model wurden fünf ausschlaggebende Domänen für ein 15-stündiges Basis-Ausbildungsprogramm identifiziert (Hinrichsen, Emery-Tiburcio, Gooblar \& Molinari, 2018). Auf Grundlage einer Befragung von 149 erfahrenen Gerontopsycholog_innen erstellten die Autor_innen eine Empfehlung (Tab. 1), welche Themen wichtiger als andere erscheinen und wie viel Lehrzeit auf die jeweiligen Domänen verteilt werden sollte (Hinrichsen et al., 2018).

Eine erste Bestandsaufnahme über die Anzahl an Ausbildungsstunden zur Psychotherapie im höheren Lebensalter (PihL) an deutschen, psychotherapeutischen Ausbildungsinstituten (Verhaltenstherapie, tiefenpsychologisch fundierte und analytische Psychotherapie) legten Kessler, Agines, Schmidt und Mühlig (2014) vor. Von den 103 teilnehmenden Instituten boten 57 Institute (55.3\%) PihLInhalte im Rahmen der Ausbildung zur/m Psychotherapeutin/en an, davon 25 mit dem Ausbildungsschwerpunkt Verhaltenstherapie. Im Durchschnitt machten PihL-Inhalte mit 12.7 Stunden im Rahmen der nach dem Psychotherapeutengesetz geforderten umfassenden theoretischen Ausbildung von 600 Stunden einen Anteil von $2.2 \%$ aus. Von den übrigen 46 Instituten, die zu dem Zeitpunkt keine gerontopsychologischen Veranstaltungen anboten, planten 11 Institute sicher (sowie 23 Institute vielleicht), in den nächsten drei Jahren gerontopsychologische Inhalte in die Curricula aufzunehmen. In der Studie wurde allerdings nur die Quantität, nicht jedoch die konkreten Lehrinhalte ermittelt.

\section{Ziel der Studie}

Um ein aktualisiertes, konkretes Bild von Quantität und Inhalten der Seminare zum Thema PihL an universitären und privaten Ausbildungsinstituten in Deutschland mit dem Schwerpunkt Verhaltenstherapie zu bekommen, wurden Leitungen von Ausbildungsinstituten und ggf. Do- zierende befragt. Dies ist wichtig, um abzubilden, wie die neue Generation der Psychotherapeut_innen in Bezug auf die psychotherapeutische Arbeit mit älteren Menschen ausgebildet ist. Außerdem sind die Ergebnisse vor dem Hintergrund der aktuellen Aus- bzw. Weiterbildungsreform für Psychotherapeut_innen im Rahmen des Psychotherapiestudiums und in der postgradualen Weiterbildung essenziell, um konkrete Weiterbildungsvorschläge formulieren zu können.

\section{Methode}

\section{Stichprobe und Ablauf}

Im Frühjahr 2018 wurden nach Sichtung aller verhaltenstherapeutischer Ausbildungsinstitute über die Listen der Landespsychotherapeutenkammern (http://www.bptk.de/ bptk/landeskammern.html) und des Verbunds universitärer Ausbildungsgänge für Psychotherapie (https://www. unith.de/) 101 für die Bestandsaufnahme in Frage kommende Institute identifiziert. Ein mit Hilfe von SocSci Survey erstellter Fragebogen wurde dann im Auftrag der Interessensgruppe „Klinische Gerontopsychologie und Psychotherapie im höheren Lebensalter" der Fachgruppe „Klinische Psychologie und Psychotherapie“ der Deutschen Gesellschaft für Psychologie (DGPs) an die jeweiligen Institutsleitungen geschickt. Die Institute wurden über die Ziele der Studie aufgeklärt, die Teilnahme erfolgte auf freiwilliger Basis. Um die Rücklaufquote zu erhöhen, wurden die Institute bei Bedarf mehrmals kontaktiert. Zusätzlich wurde in einem zweiten Schritt über die Institute Kontakt zu den Dozierenden aufgenommen, die Seminare zu PihL anboten. Die Fragebögen stehen im Elektronischen Supplement 1 und 2 zur Verfügung. 


\section{Fragebogen für die Institutsleitungen}

Zunächst wurde durch einzelne Fragen sichergestellt, dass das Institut einen verhaltenstherapeutischen Schwerpunkt besitzt und eine Ausbildung zum/zur psychologischen Psychotherapeuten_in (und nicht nur Kinder- und Jugendlichenpsychotherapeuten_in) anbietet. Dann wurde erfragt, ob Unterrichtseinheiten zu PihL im Rahmen des 600 Stunden Curriculums (nach der PsychTh-APrV) angeboten werden. Falls nicht, wurde erfragt, ob in den nächsten drei Jahren entsprechende Theoriestunden geplant seien. Weiter wurde das grundsätzliche Interesse bzw. Gründe für das fehlende Angebot erfragt, sowie benötigte Ressourcen. Bot das jeweilige Institut bereits Theoriestunden zu PihL an, wurde nach der bisherigen Dauer jenes Angebotes gefragt, sowie nach der Anzahl der Theoriestunden. Erfragt wurden zudem die Ausbau- oder Verringerungsplanung dieser Themen in den nächsten drei Jahren, sowie der Umfang von Theoriestunden zu Kindern und Jugendlichen in der Ausbildung zum/zur psychologischen Psychotherapeuten_in. Als offene Frage wurde nach den Gründen für das Theorieangebot zu PihL gefragt.

\section{Fragebogen für die Dozierenden}

Es wurde eingangs nach Berufsabschluss, Weiterbildungsqualifikation und Berufserfahrung (Dauer) gefragt. Um einen Überblick über die konkreten Ausbildungsinhalte der Seminare zu PihL zu erhalten, wurden die Dozierenden gebeten, anzugeben, ob bestimmte Inhalte einer vorgegebenen Themencheckliste (s. elektronisches Supplement 3) in ihren PihL-Seminaren vorkommen (ja/nein) ${ }^{1}$. Wenn ein Inhalt aus der Themencheckliste in einer Veranstaltung thematisiert wurde, wurde ein Kreuz an die entsprechende Unterfacette der Themencheckliste gesetzt. Die übergeordneten Themenbereiche (bspw. 4.1 „Diagnostik...") wurde dann automatisch angekreuzt, wenn eine zugehörige Unterfacette (bspw. 4.1.1 ,...kognitiver Leistungsfähigkeit") thematisiert wurde.

Die Themencheckliste wurde im Wesentlichen auf der Grundlage der „Basiswissens-“ und „Einstellungs-“ Domänen des Pikes Peak Model (Knight et al., 2009) erstellt ${ }^{2}$. Zusätzlich wurde die Themencheckliste mit Ergänzungen aus den Inhaltsverzeichnissen unterschiedlicher geron- topsychologischer Lehrbücher (Arolt \& Kersting, 2010; Forstmeier \& Maercker, 2008; Godde, Voelcker-Rehage \& Olk, 2016; Heuft, Kruse \& Radebold, 2006; Maercker, 2015; Martin \& Kliegel, 2014; Oswald, Gatterer \& Fleischmann, 2008; Peters, 2011; Steidl \& Nigg, 2014; Zank, Peters \& Wilz, 2010) komplementiert.

\section{Statistische Analysen}

Die Daten wurden mittels SPSS (Statistical Package for the Social Sciences, IBM) deskriptivstatistisch analysiert. Es wurden Mittelwerte, Standardabweichungen und (prozentuale) Häufigkeiten gebildet. Die Antworten auf die offenen Fragen zu Gründen und benötigten Ressourcen für Theorieseminare zu PihL wurden nach Häufigkeiten zusammengefasst.

\section{Ergebnisse}

\section{Quantitative Ergebnisse zu gerontopsychologischen Theorieseminaren}

Insgesamt nahmen an der Studie von 101 kontaktierten Instituten 48 Institute (47.5\%) teil. Von diesen 48 verhaltenstherapeutischen Instituten gaben 35 Institute (72.9\%) an, Unterrichtseinheiten zu PihL im Rahmen des 600stündigen Curriculums anzubieten. Drei Institute gaben dabei Veranstaltungstitel an, die unter anderem PihL-Inhalte thematisieren (bspw. Titel: „Entwicklungspsychopathologie“), ein Institut gab keinen Veranstaltungstitel an. Nach Prüfung der konkreten Veranstaltungstitel ließen sich letztlich 31 Institute mit spezifischen Seminaren zu PihL identifizieren, was etwa $64.6 \%$ von 48 teilnehmenden Instituten entspricht. Diese Seminare werden im Durchschnitt (bis 2018) seit 9.06 Jahren $(S D=6.39)$ angeboten (Tab. 2). Der durchschnittliche zeitliche Umfang von Seminaren zu PihL liegt bei 13.03 Stunden $(S D=5.32)$. Dies entspricht bei 600 Theoriestunden einem Anteil von $2.17 \%$. Von den 13.03 Stunden sind im Durchschnitt 10.43 Stunden $(S D=5.32, n=30)$ verpflichtend im 600-stündigen Curriculum (1.74\%). Der Umfang der Theoriestunden zu Kindern und Jugendlichen im Rahmen der Ausbildung zum/zur psychologischen Psy-

\footnotetext{
Alternativ hatten die Dozierenden die Möglichkeit, ihren Seminarplan samt Präsentationsmaterialien der Studienleitung zur Verfügung zu stellen. Diese wurden dann von zwei Kodiererinnen in einem Konsensverfahren entlang der Themencheckliste ausgefüllt (Kappa $=.662$ für 6 Themenchecklisten).

2 Aufgrund inhaltlicher Differenzen wurde der Wissensbereich „andere Dienstleistungen“ aus der Basiswissensdomäne „Interventionen bei Älteren und andere Dienstleistungen“ des Pikes Peak Model (Knight et al., 2009) extrahiert und für unsere Untersuchung in eine eigene Domäne umgewandelt.
} 
Tabelle 2. Ausbildungsinstitute mit Seminaren zu Psychotherapie im höheren Lebensalter

\begin{tabular}{lcccc}
\hline & $N$ & $M$ & $S D$ & Anteil an $600 \mathrm{~h}$ \\
\hline Angebot seit wann? & 31 & $9.06 \mathrm{a}$ & 6.39 & 5.32 \\
Zeitlicher Umfang & 31 & $13.03 \mathrm{~h}$ & 5.32 & $1.74 \%$ \\
Verpflichtender Umfang & 30 & $10.43 \mathrm{~h}$ & 11.54 & $3.20 \%$ \\
Zeitlicher Umfang KJ & 28 & $19.18 \mathrm{~h}$ & Nein & Weiß nicht/keine Angabe \\
\hline & $\mathrm{N}$ & $\mathrm{Ja}$ & $45.16 \%$ & $32.26 \%$ \\
Ausbau geplant? & 31 & $22.58 \%$ & $96.77 \%$ & $3.23 \%$ \\
\hline
\end{tabular}

Anmerkungen. $N$ = Stichprobengröße; $M$ = Mittelwert; SD = Standardabweichung; $K J$ = Kinder \& Jugendliche

chotherapeuten_in beträgt im Durchschnitt 19.18 Stunden $(S D=11.54, n=28) .22 .58 \%$ der 31 Institute planen in den nächsten drei Jahren einen Ausbau hinsichtlich der Seminare zu PihL, $45.16 \%$ verneinten dies, $32.26 \%$ wussten es nicht. Kein Institut plant eine Verringerung der Inhalte in den nächsten drei Jahren.

Von den 13 Instituten, die kein Seminar zu PihL anbieten, planen $30.8 \%$ die Einführung solcher Theoriestunden in den nächsten drei Jahren mit einem durchschnittlichen Stundenumfang von 13.5 Stunden $(S D=3.0), 23.1 \%$ planen keine Einführung solcher Inhalte, $38.5 \%$ sind unentschlossen, ein Institut machte keine weiteren Angaben. Auf einer vierstufigen Likert-Skala $(1=$ nicht, $2=$ wenig, $3=$ etwas, $4=$ sehr) gaben die Institute einerseits mit einem Durchschnitt von $3.25(S D=0.62, \mathrm{n}=12)$ an, an einer Einführung von PihL-Themen etwas interessiert zu sein, sowie andererseits mit einem Durchschnitt von 3.08 $(S D=0.67, \mathrm{n}=12)$, die Einführung solcher Inhalte für etwas wichtig zu halten.

\section{Gründe und Ressourcen}

Bei den 31 Instituten, die bereits Seminare zu PihL anbieten, wurden am häufigsten die folgenden drei Gründe benannt: 1) Der demographische Wandel bringt zunehmend mehr ältere Patient_innen hervor, einhergehend mit einem gestiegenen Bedarf an Psychotherapie. 2) Verweis auf spezifische Themen und Kompetenzen in der Behandlung Älterer. 3) Die Notwendigkeit der Befähigung zur Behandlung aller Altersgruppen (wie im Psychotherapeutengesetz beschrieben).

Von den 12 Instituten, die keine Seminare zu PihL anbieten, wurden hierfür die folgenden drei Gründe am häufigsten benannt: Vorrang anderer Themen, strukturelle Ursachen (bspw. kein verfügbares Seminarkonzept) und der Mangel an passenden Dozierenden. Als benötigte Ressource benannten 7 der 12 Institute kompetente Dozierende.

\section{Inhalte der Seminare zu Psychotherapie im höheren Lebensalter}

Von den 31 Instituten mit einem spezifischen Seminar zu PihL konnten bei 19 Instituten (61.3\%) inhaltliche Detailauskünfte durch mindestens einen Dozierenden eingeholt werden. 22 vorliegende Themenchecklisten wurden zur Hälfte selbstständig durch die jeweiligen Dozierenden und zur Hälfte durch zwei unabhängige Kodiererinnen auf der Basis der Seminarunterlagen ausgefüllt. Von den 22 Themenchecklisten wurden 6 ausgeschlossen, da die Veranstaltungen Themen zu PihL nur nebensächlich behandelten. Für die Bestandsaufnahme wurden letztlich 16 ausgefüllte Themenchecklisten untersucht. In zwei Fällen bezog sich die Themencheckliste auf die gleiche Veranstaltung. Diese wurden doppelt berücksichtigt, da sie an unterschiedlichen Instituten angeboten wurden. Die 16 spezifischen Seminare zu PihL wurden von 14 verschiedenen Dozierenden gehalten. Diese setzten sich aus 2 Fachärzt_innen für Psychiatrie und Psychotherapie, 11 psychologischen Psychotherapeut_innen, sowie einer Psychologin ohne Psychotherapieausbildung zusammen. Sechs Dozierende gaben als Zusatzqualifikation gerontopsychologische Zertifikate oder Weiterbildungen an, drei verwiesen auf Forschungstätigkeiten in diesem Bereich. Alle bis auf eine Dozentin berichteten praktische Erfahrung in der psychotherapeutischen Behandlung von Menschen im höheren Lebensalter, im Durchschnitt 18.69 Jahre $(S D=8.65)$.

In Tabelle 3 ist die Häufigkeitsverteilung der Themenschwerpunkte (erster Hierarchieordnung) in den Seminaren zu PihL dargestellt. Eine feinere Aufschlüsselung der Themenschwerpunkte und deren Häufigkeitsverteilung in den Theorieseminaren sind dem Elektronischen Supplement 3 zu entnehmen.

In gut zwei Drittel der Seminare wird von Domäne 1 „Der/die Therapeut_in“ der „Einfluss eigener Einstellungen und Altersstereotype auf die Behandlung Älterer" besprochen. 
Tabelle 3. Prozentuale Verteilung der Themenschwerpunkte in den Seminaren $(n=16)$

Themen

1. Der/die Therapeut/in

56.3

1.1 Einfluss eigener Einstellungen und Altersstereotype auf die Behandlung Älterer

1.2 Interaktion altersspezifischer Einstellungen mit anderen Einstellungen 43.8

2. Altern und die ältere Bevölkerung

2.1 Klärung grundlegender Begriffe

2.2 Demographische und versorgungsbezogene Aspekte

93.8

2.3 Modelle und Forschungsmethoden zum Verständnis des Alterungsprozesses 87.5

2.4 „Normales“ Altern 75

2.5 Vielgestaltigkeit des Alterungsprozesses in Abhängigkeit soziokultureller Faktoren 50

3. Grundlage klinischer Praxis mit Älteren 70.3*

3.1 Neurologische Grundlage des Alterns, deren Einfluss auf kognitive Veränderungen und deren Implikationen für klinische 37.5 Interventionen bei Älteren

3.2 Funktionelle Veränderungen im Alter und deren Auswirkungen auf den Alltag 75

3.3 Psychopathologie im mittleren und späten Erwachsenenalter 100

3.4 Häufige medizinische Erkrankungen bei Älteren 62.5

3.5 Häufige Behandlungsanlässe und -themen, Konflikte, Krisen und Belastungen Älterer 68.8

3.6 Pflegende Angehörige bzw. Pflege 62.5

3.7 Bewältigung von Trauerverlusten $\quad 68.8$

3.8 Entwicklungsaufgaben/Verluste 87.5

4. Diagnostik bei Älteren 53.2*

4.1 Diagnostik... (kog. Leistungsfähigkeit, spez. psych. Störungen, soziale Beziehungen, usw.) 93.8

4.2 Theorien und Forschung zu psychologischer Diagnostik bei Älteren 12.5

4.3 Grenzen der Nutzung von Diagnoseinstrumenten, die für Jüngere konzipiert wurden 31.3

4.4 Anpassung der Diagnostik (z. B. Instrumente, Prozess, Interpretation, ...) an Ältere 81.3

4.5 Einholen Informationen Dritter (z. B. von Angehörigen) 56.3

4.6 Einfluss von Umweltfaktoren auf diagnostischen Prozess und Ergebnisse bei Älteren 18.8

4.7 Differentialdiagnostik psychischer Störungen im Alter 81.3

4.8 Kommunikation der Ergebnisse im Gespräch 50

5. Interventionen bei Älteren 50*

5.1 Theoretischer Hintergrund, Forschungsgrundlage, Anwendung verschiedener Interventionsmethoden 100

5.2 Pharmakologische Behandlung psychischer Störungen bei Älteren 50

5.3 Schaffen eines Bewusstseins für die Bandreite potentieller Klienten für psychologische 37.5 Interventionen sowie angemessene Interventionsstrategien für diese

5.4 Modelle und Methoden interdisziplinärer Zusammenarbeit 31.3

5.5 Ethische und rechtliche Grundlagen für die psychologische Behandlung Älterer $\quad 43.8$

5.6 Besondere Aspekte der Psychotherapie Älterer $\quad 87.5$

5.7 Besonderheiten verschiedener therapeutischer Settings 62.5

5.8 Umgang mit schwierigen Behandlungssituationen 31.3

5.9 Informationen zu therapeutischen Weiterbildungsangeboten 6.3

6. Andere Dienstleistungseinrichtungen 31.3*

6.1 Hintergrundwissen über Dienstleistungen / Einrichtungen, die Ältere typischerweise aufsuchen/in denen sie leben 43.8

6.2 Überweisung zu/Empfehlung dieser Dienstleistungen (Tagespflege, betreutes Wohnen etc.) 31.3

6.3 Veränderung der Gesundheits- und Sozialversorgungssysteme 18.8

Anmerkungen. *Gemittelte Prozentzahl der jeweiligen Unterfacetten. 
In Domäne 2 „Alter und die ältere Bevölkerung“ werden am häufigsten ,demographische und versorgungsbezogene Aspekte“ (93.8\%) thematisiert, sowie "Modelle und Forschungsmethoden zum Verständnis des Alterungsprozesses“ (87.5\%) und das "normale' Altern" (75\%).

Die „Psychopathologie im mittleren und späten Erwachsenenalter“ (100\%), „Entwicklungsaufgaben und Verluste“ (87.5\%) und „Funktionelle Veränderungen im Alter“ (75\%) werden in Domäne 3 „Grundlagen klinischer Praxis mit Älteren“ am häufigsten gelehrt. „Neurologische Grundlage des Alterns" werden mit einem geringeren Anteil (37.5\%) besprochen.

In Domäne 4 „Diagnostik bei Älteren“ werden sehr häufig konkrete diagnostische Kompetenzen vermittelt (93.8\%). Lediglich in knapp einem Drittel der Seminare wird auf die „Grenzen der Nutzung von Diagnoseinstrumenten" bei älteren Menschen eingegangen, hingegen wird dafür in $81.3 \%$ der Seminare die „Anpassung der Diagnostik an Ältere" thematisiert. Häufig wird zudem die „Differentialdiagnostik psychischer Störungen im Alter" gelehrt (81.3\%).

In allen Seminaren werden Interventionen der PihL bei unterschiedlichen psychischen Erkrankungen thematisiert (Unterfacette in Domäne 5 „Interventionen bei Älteren“). Häufig kommen zudem „besondere Aspekte der Psychotherapie Älterer" (bspw. Kommunikation, therapeutische Beziehung) vor (87.5\%).

Im Vergleich mit den anderen Domänen werden spezifische Kompetenzen am wenigsten häufig in Domäne 6 „Andere Dienstleistungseinrichtungen“ vermittelt (31.3\%).

\section{Diskussion}

Der Anteil von Ausbildungsinstituten mit dem Schwerpunkt Verhaltenstherapie, die Seminare zu PihL in ihrer Theorieausbildung anbieten, liegt ähnlich wie in der vorangegangenen Studie von Kessler et al. (2014) bei etwa $64 \%$. Eine kleine Veränderung zeigt sich allerdings mit Blick auf die absoluten Zahlen. Waren es bei Kessler et al. (2014) noch 25 Institute mit dem Schwerpunkt Verhaltenstherapie, die Veranstaltungen zu PihL anbieten, so ließen sich nun 31 Institute identifizieren, die spezifische Veranstaltungen zu PihL anbieten.

Dabei entspricht der zeitliche Umfang von etwa 13 Unterrichtsstunden im Rahmen des gesamten Theoriecurriculums einem Anteil von 2.2\%, was keine Veränderung im Vergleich zum Anteil bei Kessler et al. (2014) darstellt, wobei sich letzterer auf Ausbildungsinstitute aller Psychotherapieverfahren bezieht. Da in der vorliegenden Untersuchung keine Daten von psychodynamischen Aus- bildungsinstituten vorliegen, können darüber keine Aussagen getroffen werden. Allerdings würden wir mit Blick auf die Ergebnisse von Kessler et al. (2014), die Ausbildungsinstitute aller Psychotherapieverfahren berücksichtigten, keine signifikanten Unterschiede zwischen den Therapieverfahren hinsichtlich der Quantität gerontologischer Unterrichtsstunden erwarten. Mit Blick auf den zeitlichen Umfang von Theorieinhalten über Kinder und Jugendliche (19.2 Stunden) im Rahmen der Ausbildung zum/zur psychologischen Psychotherapeuten_in zeigt sich, dass auf die Betrachtung älterer Menschen im Vergleich weniger Aufmerksamkeit gelegt zu werden scheint.

Die in der Einleitung dargelegten Gründe für mehr Lehrinhalte zu PihL in der psychotherapeutischen Ausbildung fanden sich größtenteils auch in den Rückmeldungen der Institute wieder, die bereits solche Veranstaltungen anbieten. Dies verdeutlicht nochmals besonders, dass sowohl im wissenschaftlichen Diskurs als auch in der klinischen Praxis die absolute Notwendigkeit für die spezifische Befähigung (angehender) Psychotherapeut_innen zur Behandlung älterer Menschen gesehen wird. Dabei gibt es nicht nur in Deutschland, sondern auch beispielsweise in den USA Forderungen nach mehr Weiterbildung zu PihL für klinisch tätige PsychologInnen, um dem steigenden Bedarf gerecht werden zu können (Moye et al., 2019).

In der vorliegenden Untersuchung werden in den Seminaren ähnlich wie bei Hinrichsen et al. (2018) sowohl allgemeine, entwicklungsbezogene Grundlagen des Alterns als auch besondere psychopathologische Phänomene im späteren Erwachsenenleben als bedeutsam angesehen. Ferner werden sowohl altersspezifische, diagnostische Kompetenzen als auch spezifische Interventionsmethoden bei psychischen Störungen (bspw. die Lebensrückblicktherapie) häufig thematisiert. Weniger präsent scheinen im Gegensatz zu den Empfehlungen von Hinrichsen et al. (2018) in den Seminaren neurobiologische Grundlagen, sowie psychopharmakologische Lehrinhalte zu sein. Ausbaufähig scheint vor dem Hintergrund der vorliegenden Untersuchung die Wissensvermittlung von altersspezifischen Dienstleistungseinrichtungen im Bereich der Altenhilfe, da sich gerade die interdisziplinäre Vernetzung von Psychotherapeut_innen, Hausärzt_innen und Pflegekräften positiv auf die Behandlung auswirken kann (Kessler \& Tegeler, 2018). Weiterhin wird das Thema Pflegende Angehörige von älteren Menschen bislang in den Seminaren zu PihL eher wenig thematisiert und sollte perspektivisch in die Curricula aufgenommen werden (Wilz, Schinköthe \& Kalytta, 2015; Wilz \& Pfeiffer, 2019). Ein obligatorischer Bestandteil von Aus- und Weiterbildung sollte auch darin bestehen, Psychotherapeut_innen dabei zu unterstützen, eine differenzierte Sicht auf das Alter zu entwickeln, da unreflektierte, altersstereotype Gedanken des/der Therapeuten_in den Therapiepro- 
zess negativ beeinflussen könnten (Knight, 2018). Verfügen Psychotherapeut_innen über ein realistisches Altersbild, dann sind sie in der Lage, die individuellen Ressourcen, Präferenzen und Defizite ihrer Patient_innen zu erkennen, und sie können mit ihnen in den Therapien an deren Altersbildern arbeiten (Kessler, 2021).

\section{Limitationen}

Sowohl die Rücklaufquote bei den Ausbildungsinstituten (47.5\%) als auch die Stichprobengröße der Dozierenden sind als eher gering zu bewerten, was eine Generalisierbarkeit der Ergebnisse erschwert. Zudem wurde bei der Kodierung der Themenschwerpunkte kein einheitliches System genutzt, was die Reliabilität schwächt. Weiterhin sollte berücksichtigt werden, dass bei einer solchen Studie wohlmöglich eher jene Institute teilnehmen, die mehr Interesse an PihL haben. Das würde bedeuten, dass die Ausbildungssituation in verhaltenstherapeutischen Instituten zum Thema PihL wohlmöglich weniger weit fortgeschritten ist, als die aktuelle Bestandsaufnahme zeigt. Andererseits wurde nicht untersucht, inwieweit gerontopsychologische Inhalte auch in anderen Seminaren (bspw. Störungslehre) in Teilen aufgegriffen werden, was wiederum zu einer Unterschätzung der Lehrinhalte zu PihL im Rahmen der Psychotherapieausbildung führen könnte.

\section{Ausblick und Implikationen für die neuen Psychotherapiestudiengänge}

In der neuen Approbationsordnung für Psychotherapeut_innen (PsychThApprO) vom 4. März 2020 (Bundesgesetzblatt, 2020) ist sowohl im geplanten Bachelor- als auch Masterstudium das höhere Lebensalter an diversen Stellen explizit erwähnt. Dabei wird es insbesondere wichtig sein, klinisch-praktische Erfahrungen mit älteren Patient_innen in den Curricula mit einfließen zu lassen, da dies das Interesse an der klinischen Arbeit mit älteren Menschen fördert (Koder \& Helmes, 2008).

1. Gerontopsychologie ist im Bachelorstudiengang leider nicht als Modul vorgesehen. Es sollte daher seitens der Dozierenden darauf geachtet werden, dass Alter(n) als Querschnittsthema zu berücksichtigen ist, etwa in der Lehre in den Bereichen Grundlagen der Psychologie/Medizin, Störungslehre, Diagnostik, Allgemeine Verfahrenslehre der Psychotherapie, präventive und rehabilitative Konzepte psychotherapeutischen Handelns. Die Entwicklungspsychologie sollte als Lebensspannenpsychologie betrachtet werden.

2. Im Masterstudiengang ist erfreulicherweise zumindest theoretisch eine vertiefte theoretische Wissensvermittlung zu PihL vorgesehen, wenn es bspw. in $\$ 10$ heißt, dass in der „Berufsqualifizierenden Tätigkeit II - vertiefte Praxis der Psychotherapie" von den 15 ECTSPunkten „mindestens 5 ECTS-Punkte auf den Wissensbereich Ausübung von Psychotherapie bei Erwachsenen und älteren Menschen" (S. 451) entfallen sollen. Die praktisch-klinische Erfahrung mit älteren Patient_innen sollte anteilig gemäß $₫ 18$ im Modul „Berufsqualifizierende Tätigkeit III - angewandte Praxis der Psychotherapie“ erfolgen, wenn es heißt, dass „bei mindestens zehn Patientinnen und Patienten verschiedener Alters- und Patientengruppen“" (S. 453) beispielsweise Anamnesen $\mathrm{zu}$ erstellen oder an psychotherapeutischen Sitzungen teilzunehmen ist. Es sollte seitens der Hochschulen darauf geachtet werden, dass diese Vorgaben auch in der Lehrpraxis umgesetzt werden, und nicht als Randthema behandelt wird. Dies setzt voraus, dass sich Hochschulen um fachkompetentes Lehrpersonal mit spezifischer gerontopsychologischer Qualifikation bemühen sowie um die Kooperation mit Einrichtungen, die ältere Patient_innen behandeln.

3. Die auch weiterhin bestehende, psychotherapeutische postgraduale Weiterbildung nach dem Studium sollte vor dem Hintergrund eines theoretisch-konzeptionellen Rahmens, der sich auf die hier dargestellten Themenschwerpunkte (Tabelle 3) bezieht, theoretische und praktische Inhalte zu PihL vermitteln. Als Handreichung für die Aus- und Weiterbildungsinstitute plant daher die Interessengruppe „Klinische Gerontopsychologie und Psychotherapie im höheren Lebensalter" eine fokussierte Ausarbeitung des theoretisch-konzeptionellen Rahmens auf Grundlage der hiesigen Bestandsaufnahme.

\section{Elektronische Supplemente (ESM)}

Die Elektronischen Supplemente sind mit der OnlineVersion dieses Artikels verfügbar unter https://doi.org/ 10.1026/1616-3443/a000593

ESM 1. Fragebogen Institutsleitung (ESM1.Fragebogen Institutsleitung.docx)

ESM 2. Fragebogen Dozenten (ESM2.Fragebogen Dozenten.docx)

ESM 3. Gesamte Themencheckliste (ESM3.Themencheckliste.xlsx). Die Tabelle enthält die prozentuale Verteilung der Themenschwerpunkte in den Seminaren $(n=16)$ 


\section{Literatur}

American Psychological Association. (2014). Guidelines for Psychological Practice With Older Adults. American Psychologist, 69 (1), 34-65. https://doi.org/10.1037/a0035063

Arolt, V. \& Kersting, A. (2010). Psychotherapie in der Psychiatrie. Berlin, Heidelberg: Springer.

Ayers, C. R., Sorrell, J. T., Thorp, S. R. \& Wetherell, J. L. (2007). Evidence-Based Psychological Treatments for Late-Life Anxiety. Psychology and Aging, 22 (1), 8-17. https://doi.org/10. 1037/0882-7974.22.1.8

Boerner, R. J. (2004). Angst im Alter - Epidemiologie, Diagnostik und therapeutische Optionen. Fortschritte der Neurologie, Psychiatrie, 72, 564-573. https://doi.org/10.1055/s-2004-818 530

Böttche, M., Kuwert, P. \& Knaevelsrud, C. (2012). Posttraumatic stress disorder in older adults: an overview of characteristics and treatment approaches. International Journal of Geriatric Psychiatry, 27, 230 - 239. https://doi.org/10.1002/gps.2725

Bundesgesetzblatt. (2020). Approbationsordnung für Psychotherapeutinnen und Psychotherapeuten (PsychThApprO) vom 4. März 2020. Bundesgesetzblatt Jahrgang 2020 Teil I Nr. 11, ausgegeben zu Bonn am 12. März 2020. Bundesanzeiger Verlag.

Cuijpers, P., Karyotaki, E., Pot, A. M., Park, M. \& Reynolds, C. F. (2014). Managing depression in older age: psychological interventions. Maturitas, 79, 160 -169. https://doi.org/10.1016/j.ma turitas.2014.05.027

Forstmeier, S. \& Maercker, A. (2008). Probleme des Alterns (Fortschritte der Psychotherapie). Göttingen: Hogrefe.

Forstmeier, S. \& Roth, T. (2018). Kognitive Verhaltenstherapie für Patienten mit leichter Alzheimer-Demenz und ihre Angehörigen. Berlin: Springer.

Godde, B., Voelcker-Rehage, C. \& Olk, B. (2016). Einführung Gerontopsychologie. München: Ernst Reinhardt.

Gould, R. L., Coulson, M. C. \& Howard, R. J. (2012). Efficacy of Cognitive Behavioral Therapy for Anxiety Disorders in Older People: A Meta-Analysis and Meta-Regression of Randomized Controlled Trials. Journal of the American Geriatrics Society, 60, 218-229. https://doi.org/10.1111/j.1532-5415.2011.03824.x

Gühne, U., Luppa, M., König, H.-H., Hautzinger, M. \& Riedel-Heller, S. (2014). Ist Psychotherapie bei depressiven Erkrankungen im Alter wirksam? Ein systematischer Überblick. Psychiatrische Praxis, 41, 415-423. https://doi.org/10.1055/s-0034-1370113

Hall, J., Kellett, S., Berrios, R., Bains, M. K. \& Scott, S. (2016). Efficacy of Cognitive Behavioral Therapy for Generalized Anxiety Disorder in Older Adults: Systematic Review, Meta-Analysis and Meta-Regression. The American Journal of Geriatric Psychiatry, 24, 1063-1073. https://doi.org/10.1016/j.jagp.2016.06.006

Heuft, G., Kruse, A. \& Radebold, H. (2006). Lehrbuch der Gerontopsychosomatik und Alterspsychotherapie (2. Auflage). München: Ernst Reinhardt.

Hinrichsen, G. A., Emery-Tiburcio, E. E., Gooblar, J. \& Molinari, V. A. (2018). Building foundational knowledge competencies in professional geropsychology: Council of Professional Geropsychology Training Programs (CoPGTP) recommendations. Clinical Psychology, Science and Practice, 25, e12236. https://doi.org/ $10.1111 /$ cpsp.12236

Jacobi, F., Höfler, M., Strehle, J., Mack, S., Gerschler, A., Scholl, L., Busch, M. A., Maske, U., Hapke, U., Gaebel, W., Maier, W., Wagner, M., Zielasek, J. \& Wittchen, H.-U. (2014). Psychische Störungen in der Allgemeinbevölkerung: Studie zur Gesundheit Erwachsener in Deutschland und ihr Zusatzmodul Psychische Gesundheit (DEGS1-MH). Nervenarzt, 85, 77-87. https://doi. org/10.1007/s00115-013-3961-y
Kessler, E.-M. (2021). Psychotherapeutisches Arbeiten mit alten und sehr alten Menschen. Stuttgart: Kohlhammer.

Kessler, E.-M. \& Blachetta, C. (2020). Age cues in patients' descriptions influence treatment attitudes. Aging \& Mental Health, 24, 193 -196. https://doi.org/10.1080/13607863.2018.1515889

Kessler, E.-M. \& Tegeler, C. (2018). Psychotherapeutisches Arbeiten mit alten und sehr alten Menschen. Psychotherapeut, 63, 501 - 518. https://doi.org/10.1007/s00278-018-0315-z

Kessler, E.-M. \& Peters, M. (2017). Befindet sich die Alterspsychotherapie im Aufbruch? Anmerkungen zur Entwicklung und zum aktuellen Stand. Psychotherapie im Alter, 14 (1), 7 - 16.

Kessler, E.-M., Agines, S., Schmidt, C. \& Mühlig, S. (2014). Qualifikationsmöglichkeiten im Fachgebiet Gerontopsychologie: Empirische Bestandsaufnahme. Zeitschrift für Gerontologie und Geriatrie, 47, 337-344. https://doi.org/10.1007/s00391-0130553-1

Knight, B.G. (2018). Commentary on "Building foundational knowledge competencies in professional geropsychology". Clinical Psychology, Science and Practice, 25, e12239. https://doi.org/ 10.1111/cpsp.12239

Knight, B. G., Karel, M. J., Hinrichsen, G. A., Qualls, S. H. \& Duffy, M. (2009). Pikes Peak Model for Training in Professional Geropsychology. American Psychologist, 64, 205-214. https://doi.org/ 10.1037/a0015059

Koder, D.-A. \& Helmes, E. (2008). Predictors of interest in working with older adults: a survey of postgraduate trainee psychologists. Gerontology \& Geriatrics Education, 29 (2), 158-171. https://doi.org/10.1080/02701960802223233

Maercker, A. (Hrsg.). (2015). Alterspsychotherapie und klinische Gerontopsychologie (2. Auflage). Berlin, Heidelberg: Springer.

Martin, M. \& Kliegel, M. (2014). Psychologische Grundlagen der Gerontologie (4. Auflage). Stuttgart: Kohlhammer.

Moye, J., Karel, M. J., Stamm, K. E., Qualls, S. H., Segal, D. L., Tazeau, Y. N. \& DiGilio, D. A. (2019). Workforce Analysis of Psychological Practice With Older Adults: Growing Crisis Requires Urgent Action. Training and Education in Professional Psychology, 13 (1), 46 - 55. https://doi.org/10.1037/tep0000206

Nau, S. D., McCrae, C. S., Cook, K. G. \& Lichstein, K. L. (2005). Treatment of insomnia in older adults. Clinical Psychology Review, 25, 645 -672. https://doi.org/10.1016/j.cpr.2005.04.008

Oswald, W.-D., Gatterer, G. \& Fleischmann, U. M. (2008). Gerontopsychologie: Grundlagen und klinische Aspekte zur Psychologie des Alterns. Wien: Springer.

Peters, M., Jeschke, K. \& Peters, L. (2013). Ältere Patienten in der psychotherapeutischen Praxis - Ergebnisse einer Befragung von Psychotherapeuten. Psychotherapie, Psychosomatik, Medizinische Psychologie, 63, 439 - 444. http://dx.doi.org/10.1055/ s-0033-1345117

Peters, M. (2011). Leben in begrenzter Zeit: Beratung älterer Menschen. Göttingen: Vandenhoeck \& Ruprecht.

Pinquart, M., Duberstein, P. R. \& Lyness, J. M. (2007). Effects of psychotherapy and other behavioral interventions on clinically depressed older adults: A meta-analysis. Aging \& Mental Health, 11, 645 - 657. https://doi.org/10.1080/13607860701529635

Richter, K., Kellner, S., Milosheva, L. \& Fronhofen, H. (2020). Treatment of insomnia in elderly patients. Journal of ReAttach Therapy and Developmental Diversities, 2 (2), $129-138$. https://doi. org/10.26407/2019jrtdd.1.25

Statistisches Bundesamt. (2020). Selbstmordrate in Deutschland nach Altersgruppe in den Jahren 2014 bis 2018 [Zitiert nach de. statista.com]. Verfügbar unter: https://de.statista.com/statis tik/daten/studie/318224/umfrage/selbstmordrate-in-deutsch land-nach-altersgruppe/

Statistisches Bundesamt (Destatis). (2019). Bevölkerung im Wandel: Annahmen und Ergebnisse der 14. koordinierten Bevölkerungsvorausberechnung. Verfügbar unter: https://www.desta 
tis.de/DE/Presse/Pressekonferenzen/2019/Bevoelkerung/pres sebroschuere-bevoelkerung.pdf?__blob=publicationFile

Steidl, S. \& Nigg, B. (2014). Gerontologie, Geriatrie und Gerontopsychiatrie: Ein Lehrbuch für Gesundheits- und Pflegeberufe (4. Auflage). Wien: Facultas.

Verdurmen, M. J. H., Videler, A. C., Kamperman, A. M., Khasho, D. \& van der Feltz-Cornelis, C. M. (2017). Cognitive behavioral therapy for somatic symptom disorders in later life: a prospective comparative explorative pilot study in two clinical populations. Neuropsychiatric Disease and Treatment, 13, 23312339. https://doi.org/10.2147/NDT.S141208

Wächtler, C. (2009). Suizidalität älterer Menschen - Erkennen, ernst nehmen, be-handeln. Psychotherapie, 14, 306-314.

Wilz, G. \& Pfeiffer, K. (2019). Pflegende Angehörige. Göttingen: Hogrefe Verlag.

Wilz, G., Schinköthe, D. \& Kalytta, T. (2015). Therapeutische Unterstützung für pflegende Angehörige von Menschen mit Demenz. Göttingen: Hogrefe Verlag.

Zank, S., Peters, M. \& Wilz, G. (2010). Klinische Psychologie und Psychotherapie des Alters. Stuttgart: Kohlhammer.

\section{Autorenschaften}

Die Mitautor_innen sind erreichbar unter: Franziska Martin, mar tin-franziska@gmx.de; Gabriele Wilz, gabriele.wilz@uni-jena.de; Anne Katrin Risch, anne.katrin.risch@uni-jena.de; Eva-Marie Kessler, eva-marie.kessler@medicalschool-berlin.de; Simon Forstmeier, simon.forstmeier@uni-siegen.de

\section{Förderung}

Open Access-Veröffentlichung ermöglicht durch die Universität Siegen.

Tobias Becker, M. Sc. Psych.

Entwicklungspsychologie und Klinische Psychologie der Lebensspanne (EKLIPS)

Institut für Psychologie

Universität Siegen

Adolf-Reichwein-Str. 2a

57068 Siegen

tobias2.becker@student.uni-siegen.de 